\title{
Facile Synthesis of 3-Alkoxymethyl 2(1H)-Quinolinones from the Baylis-Hillman Adducts of 2-Nitrobenzaldehydes
}

\author{
Ka Young Lee, Jeong Mi Kim, and Jae Nyoung Kim*

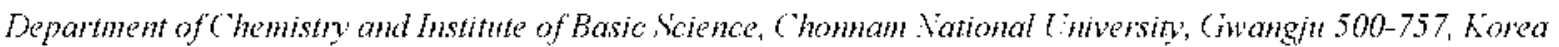 \\ Received June 7, 2002
}

Key Words : 2(1H)-Quinolinome, Bạ lis-Hillman adducts, 2-Nitrohenyaldehỵdes, Tin(II) chloride

The Baylis-Hillman reacion is a useful carbon-carbon bond-forming method from aclivaled vinyls and carbonyl compounds. ' Chemical transformation of the Baylis-Hillman adducts or their derivatives into useful helerocyclic compounds have been studied recently by us and other groups. ${ }^{2.3}$ Especially comersion of the Bay lis-Hillman adducis derived from 2-1nitrobenzaldehydes into quinoline skeleton is a useful entry for the quinoline chemistry. The 2(1H)-quinolinone ring system is found in many biologically important compounds. "Thus. the development of a new method for the synthesis of $2(1 H)$-quinolinone ring system is important until now.

Recently: we have reported on the synthesis of 3-substituled 2(1H)-quinolinone derivatives by the reduction of the Baylis-Hillnan adducts with rinc and appropriate carboxylic acid in the presence of catalytic amounts of trifluoroacelic acid (Scheme 1), ${ }^{5}$ The reaction procecded by the tandem reduction. intramolecular amide bond formation. conjugate addition of the carboxylic acid and delydration. Thus. the use of alcohol solvents under the appropriate reducing conditions might give the corresponding 3-alkoxymellyy]-substituled 2( $1 H$ )-quinolinone derivatives.

Among the various examined reduction conditions. the use of lin(II) cliloride in an alcohol solyent was found to meet<smiles>[R]C(=O)OCc1cc2ccccc2[nH]c1=O</smiles>

Scheme 1

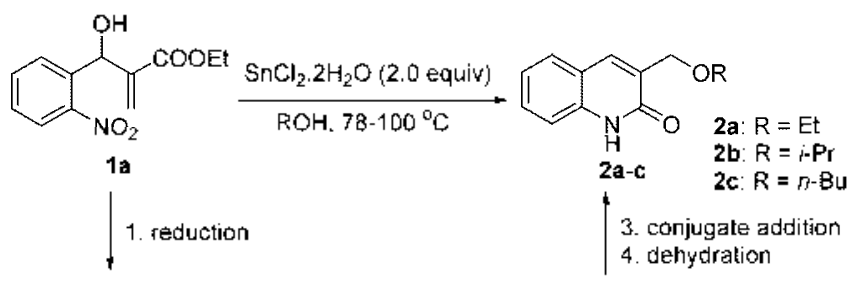<smiles>C=C(OCC)C(O)c1ccccc1N</smiles>

3

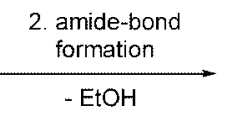<smiles>C=C1C(=O)Nc2ccccc2C1O</smiles>

4
Scheme 2 our requirement. The reaction of the Baylis-Hillman adducts 1a and tin(II) chloride dilıdrate (2,0 cquiy.) in ethanol at reflux 1emperature gave $2 a$ in $60 \%$ isolated yicld (Scheine 2 and cutry $I$ in Table 1). The same reaction in 2-propanol afforded the corresponding isopropoxy derivative $2 \mathbf{b}$ in a similar yicld (entry 2.58\%). Similarly butoxy derivative $2 \mathrm{c}$ was obtained in $n$-butanol (entry 3). The representatice results are summarized in Table 1. When we used alkoxysubstituled starting materials such as $\mathbf{1 d}$ and $1 \mathrm{e}$. somewhat

Table 1. Synthesis of 3-alkoxymethyl 2(1//)-quinolinones 2

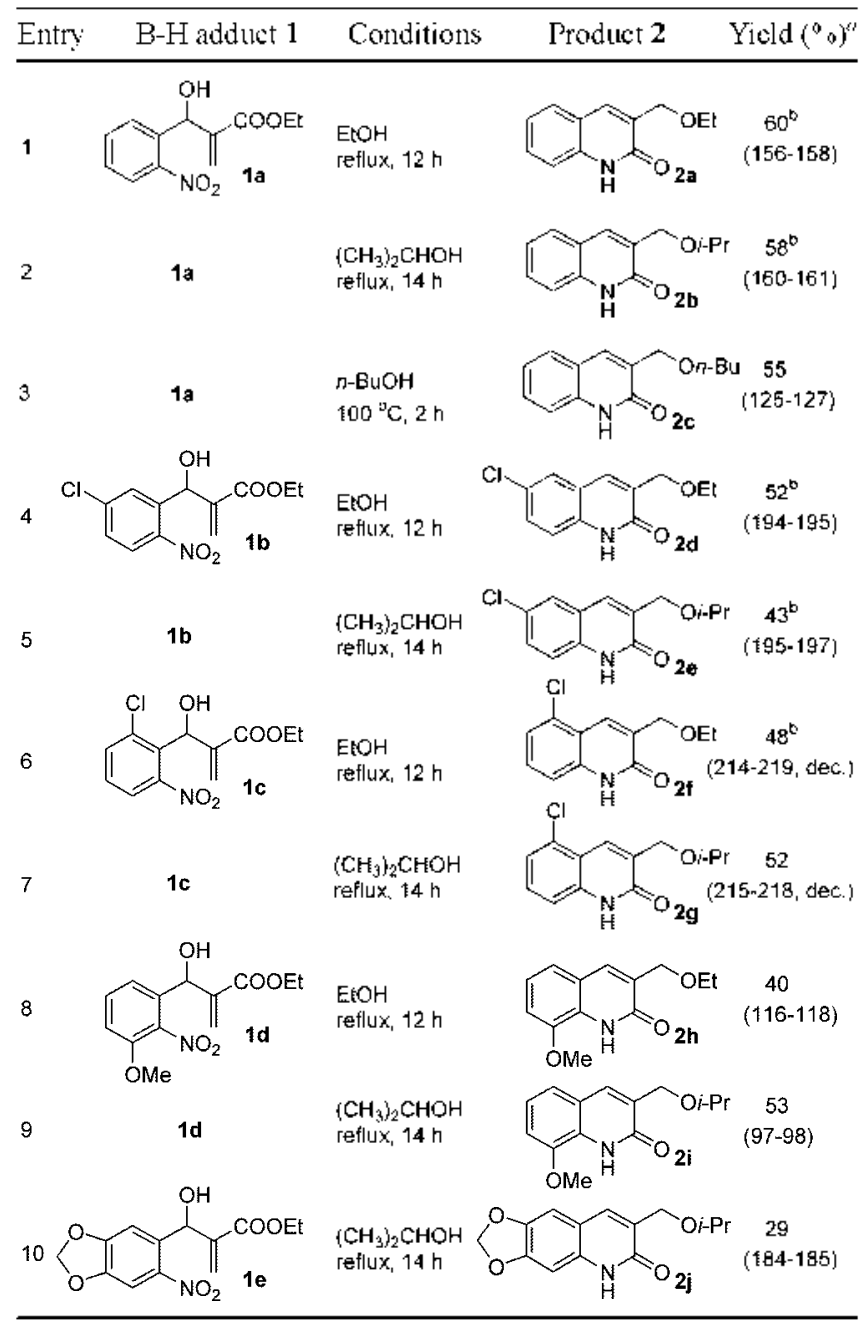

"Mp was written in parenthesis. "The comesponding benzisovazoline derivatives were obtained in $5-10^{\circ}$ y yiclds. 
<smiles>CCOC(=O)C(=O)C(O)c1cc(Cl)ccc1[N+](=O)[O-]</smiles>

1b

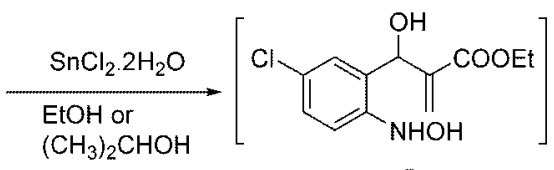

5

7

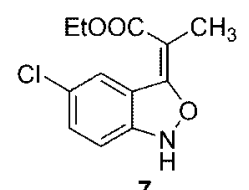

Scheme 3

lower yields of products were obtained.

The reaction mechanism is thought to be as follows as shown in Scheme 2: (1) Reduction of the nitro functionality of 1 to the amino group. (2) intramolecular amide-bond fornation and finally (3) conjugate addition of the alcohol followed by delydration.

As a side reaction benzisoxazoline derivatives were isolated in some cases. As an example, in the reaction of $\mathbf{1 b}$ (entry 4 ) we could isolate benzisoxazoline 7 in about $10 \%$ yield. This compound might be obtained from the hydroxylamine derivative 5 . which was generated by partial reduction of $1 \mathrm{~b}$ as shown in Scheme 3 . The same compound 7 was isolated when the reaction was performed in 2-propanol. The stereochemistry of the double bond of 7 was assigned as $E$ from the NOE experiment (no NOE increment of the proton at the benzene ring was observed when we irradiated the methyl peak at $\delta=2.74 \mathrm{ppm}$ ).

As a conclusion. we disclosed the facile one-pot preparation method of 3-alkoxymethyl-substituted 2(1//)-quinolinones from the Baylis-Hillman adducts of 2-nitrobenzaldehydes.

\section{Experimental Section}

All materials and solvents were of reagent grade as received from commercial sources. Baylis-Hillman adducts were prepared as reported. ${ }^{1-3}$

Typical procedure for the synthesis of $2 \mathrm{a}$ : A stirred solution of $1 \mathrm{a}(251 \mathrm{mg} .1 .0 \mathrm{mmol})$ and tin chloride dihydrate ( $450 \mathrm{mg} .2 .0 \mathrm{mmol}$ ) in ethanol $(5 \mathrm{~mL}$ ) was heated to reflux for $12 \mathrm{~h}$. After appropriate workup process and column chromatographic purification (hexane/ethyl acetate $=1: 1$ ) 2a was obtained as a white solid. $122 \mathrm{mg}(60 \%)$ : $\mathrm{mp}$ 156$158^{\circ} \mathrm{C}$ : IR ( $\left.\mathrm{KBr}\right) 3446,1663 \mathrm{~cm}^{-1}:{ }^{\prime} \mathrm{H} \mathrm{NMR}\left(\mathrm{CDCl}_{3}\right) \delta 1.34$ $($ t. $J=6.9 \mathrm{~Hz} .3 \mathrm{H}) .3 .72(\mathrm{q} . J=6.9 \mathrm{~Hz} .2 \mathrm{H}) .4 .6 \mathrm{l}(\mathrm{s} .2 \mathrm{H})$. 7.19-7.61 (m. HH). 7.91 (s, lH). 11.30 (s, lH) ${ }^{1.3} \mathrm{C}$ NMR $\left(\mathrm{CDCl}_{3}\right) \delta 15.27,66.70,67.18,115.48,120.08,122.63$. $127.75,129.83 .130 .60,135.99,137.40,162.76$ : Mass $(70$ eV) $m z$ (rel. intensity) 77 (18). 128 (29). $130(26) .159$ $(100) .172(19) .174(48) .203(\mathrm{M} .2)$. The following compounds were synthesized analogously.

3-(Isop mpoxymethyl)-(1H)-quinol-2-one (2b): $58 \%$; mp 160-161 "C: IR (KBr) 2968, 1661. $1575 \mathrm{~cm}^{-1}:{ }^{1} \mathrm{H}$ NMR $\left(\mathrm{CDCl}_{3}\right) \delta 1.31(\mathrm{~d} . J=6.0 \mathrm{~Hz} .6 \mathrm{H}) .3 .8+$ (heptet $J=6.0 \mathrm{~Hz}$.
lH). 4.62 (s. $2 \mathrm{H}), 7.18-7.60$ (m. $4 \mathrm{H}), 7.94$ (s. lH). 12.27 (s. 1H); ${ }^{13} \mathrm{C}$ NMR $\left(\mathrm{CDCl}_{3}\right) \delta 22.25,64.83,72.16 .115 .73$, 120.13. 122.53. 127.62, 129.67, 131.01. 135.84. 137.45. 163.22: MS (70 eV) $m z$ (rel. intensity) 128 (19). $146(9)$. $159(100) .174(64) .217\left(\mathrm{M}^{\prime}, 1\right)$.

3-(Butoxymethyl)-(1H)-quinol-2-one (2c): 55\%, np 125$127{ }^{\circ} \mathrm{C}:{ }^{1} \mathrm{H}$ NMR $\left(\mathrm{CDCl}_{3}\right) \delta 0.97$ (t. $\left.J=7.2 \mathrm{~Hz}, 3 \mathrm{H}\right), 1.41-$ 1.54 (m. $2 \mathrm{H}) .1 .65-1.75(\mathrm{~m} .2 \mathrm{H}) .3 .66(\mathrm{t} . J=7.2 \mathrm{~Hz} .2 \mathrm{H})$. $4.6 \mathrm{l}$ (s. 2H). 7.16-7.58 (m. HH). 7.90 (s. lH). 12.74 (s. lH); ${ }^{1.2} \mathrm{C}$ NMR $\left(\mathrm{CDCl}_{3}\right) \delta 14.00,19.43,31.87,67.40 .71 .15$. 115.96. 120.05. 122.54, 127.57, 129.76. 130.38. 136.02. 137.59. 163.44.

3-(Ethoxymethyl)-6-chloro-(1H)-quinol-2-one (2d): 52\%: mp 194-195 ${ }^{\circ} \mathrm{C}:{ }^{\prime} \mathrm{H} \mathrm{NMR}\left(\mathrm{CDCl}_{3}\right) \delta 1.34(\mathrm{t} . J=7.2 \mathrm{~Hz} .3 \mathrm{H})$. 3.72 (q. $J=7.2 \mathrm{~Hz}, 2 \mathrm{H}) .4 .59$ (s. $2 \mathrm{H}) .7 .36(\mathrm{~d} . J=8.7 \mathrm{~Hz}$. lH). 7.43 (d. $J=8.7 \mathrm{~Hz}$. $1 \mathrm{H}$ ). 7.57 (s. $\mathrm{HH}) .7 .84$ (s. $\mathrm{lH}$ ), 12.43 (s. $1 \mathrm{H}):{ }^{1.2} \mathrm{C}$ NMR $\left(\mathrm{CDCl}_{3}\right) \delta 15.27,66.81 .67 .07$. 117.23. 121.00, 126.78, 127.89, 130.03. 131.80. 134.85. 135.89. 163.03 .

3-(Isopropoxymethyl)-6-chloro-(1H)-quinol-2-one (2e): 43\%: mp 195-197 " $\mathrm{C}:{ }^{\prime} \mathrm{H}$ NMR $\left(\mathrm{CDCl}_{3}\right) \delta 1.31$ (d. $J=6.0$ Hz. $6 \mathrm{H}$ ), 3.82 (heptet, $J=6.0 \mathrm{~Hz}, 1 \mathrm{H}$ ). 4.59 (s, $2 \mathrm{H}$ ), $7.25-$ 7.58 (m. 3H). 7.87 (s. IH). 12.40 (s. lH) : ${ }^{1.3} \mathrm{C}$ NMR (DMSOd $\mathrm{d}_{6} \delta 22.21 .64 .38,71.41,116.85,120.48,125.79,126.84$. 129.60. 132.68. 133.62, 136.62, 160.86.

3-(Ethoxymethyl)-5-chloro-(1 $H)$-quinol-2-one (2f): 48\%: mp $214-219^{\circ} \mathrm{C}$ (dec): ' $\mathrm{H}$ NMR $\left(\mathrm{CDCl}_{3}\right) \delta 1.35$ (t. $J=7.2$ Hz. $3 \mathrm{H}) .3 .73(\mathrm{q} . J=7.2 \mathrm{~Hz}, 2 \mathrm{H}), 4.62(\mathrm{~s} .2 \mathrm{H}), 7.26-7.42(\mathrm{~m}$. $3 \mathrm{H}) .8 .32$ (s. IH). 12.28 (s. 1H): ${ }^{13} \mathrm{C} \mathrm{NMR}\left(\mathrm{CDCl}_{3}\right) \delta 15.28$. 66.78.67.19. 114.72. 118.00. 123.25. 130.09. 131.57. 132.31. 132.63. 138.6+. 163.00.

3-(Isopropoxymethyl)-5-chloro-(1H)-quinol-2-one (2g): 52\%: mp 215-218 " $\mathrm{C}$ (dec.): ${ }^{1} \mathrm{H}$ NMR $\left(\mathrm{CDCl}_{3}\right) \delta 1.31$ (d. $J=$ $6.0 \mathrm{~Hz}, 6 \mathrm{H}$ ), 3.83 (heptet, $J=6.0 \mathrm{~Hz}, \mathrm{lH}$ ), 4.62 (s. $2 \mathrm{H}$ ). $7.25-7 .+3(\mathrm{~m}, 3 \mathrm{H}) .8 .3+$ (s. $\mathrm{HH}) .11 .7 \mathrm{l}(\mathrm{s} . \mathrm{HH})$.

3-(Ethoxymethyl)-8-methoxy-(1H)-quinol-2-one (2h): $40 \%$, mp 116-118 ${ }^{\circ} \mathrm{C}:{ }^{\prime} \mathrm{H}$ NMR $\left(\mathrm{CDCl}_{3}\right) \delta 1.32$ (t. $J=7.2$ Hz. $3 \mathrm{H}) .3 .69$ (q. $J=7.2 \mathrm{~Hz} .2 \mathrm{H}) .3 .97$ (s. $3 \mathrm{H}) .4 .56$ (s. $2 \mathrm{H}$ ). 6.95-7.20 (m. 3H). 7.85 (s. IH) 9.34 (s. IH): ${ }^{13} \mathrm{C}$ NMR $\left(\mathrm{CDCl}_{3}\right) \delta$ 15.17. 55.91, 66.65, 67.13. 109.34, 119.51. 120.14. 122.08. 127.43, 131.52, 135.28. 1+5.31, 160.87.

3-(Isopmopoxymethyl)-8-methoxy-(1H)-quinol-2-one (2i): 53\%: mp 97-98 ${ }^{\circ} \mathrm{C}$ : 'H NMR $\left(\mathrm{CDCl}_{3}\right) \delta \mathrm{L} .28(\mathrm{~d} . J=6.0 \mathrm{~Hz}$. $6 \mathrm{H}) .3 .80$ (heptet. $J=6.0 \mathrm{~Hz} . \mathrm{lH}$ ). 3.97 (s. $3 \mathrm{H}) .4 .56$ (s. $2 \mathrm{H}$ ). 6.92-7.27 (m. 3H). 7.87 (s. 1H). 9.27 (s. $\mathrm{lH}$ ): ${ }^{1 .} \mathrm{C}$ NMR $\left(\mathrm{CDCl}_{3}\right) \delta 22.22 .55 .95,64.83,72.23 .109 .26,119.56$. 120.26. 122.13, 127.33, 132.12, 135.08. 145.35, 160.97.

3-(Isopmpoxymethyl)-6,7-methylenedioxy-(1H)-quinol2-one (2j): $2 \% \%$; mp $184-185^{\circ} \mathrm{C}:{ }^{\prime} \mathrm{H}$ NMR $\left(\mathrm{CDCl}_{i}\right) \delta 1.30$ (d. $J=6.0 .6 \mathrm{H}$ ). 3.82 (heptet. $J=6.0 \mathrm{~Hz} . \mathrm{lH}$ ). +.57 (s. $2 \mathrm{H}$ ). 6.04 (s. 2H). 6.88 (s. lH). 6.95 (s. lH). 7.80 (s. $1 \mathrm{H}$ ). $12.1+$ (s. IH): ${ }^{13} \mathrm{C}$ NMR (DMSO-d 101.69. 105.+1. 113.48, 128.01, 134.71. 135.00. 1+3.25. 149.5+. 160.85 .

Acknowledgment. This work was supported by a Korea Research Foundation Grant (KRF-2001-015-DP0326). 


\section{References and Notes}

1. (a) Basavaiah. D.: Rao. R. D.: Hyma. R. S. Tetrahedon 1996. 52. 8001. (b) Ciganek, Г. Organic Reactions; John Wiley \& Sons: New York, 1997: Vol. 51. pp 201-350. (c) Drewes, S. F.: Roos. G. II. P. Titrihedron 1988, 4t. 4653. (d) Ianger, P. Angen: Chem. Iht F. . 2000. 39. 3049 .

2. For our recent papers on the synthesis of heterocyclic compounds see. (a) Kim. J. N.: Lee. H. J.: Lee. K. Y.: Kim. H. S. Tethohedon Letf. 2001. 42. 3737. (b) Chung. Y. M.: I ce. II. J. J Iwang. S. S.: Kim. J. N. Bwll. Konem Chtm. Soc. 2001. 22. 799. (c) Kim, J. N.: Kim, J. S.: Gong. J. II.: Chung. Y. M. Trtahtedrm Lsff. $2001, d 2$. 8341.

3. (a) Kim. J. N.: Lee. K. Y.: Kim. H. S.: Kim. T. Y. Org. Lett 2000. 2. 343. (b) Kim. J. N.: Lee. K. Y.: Ham. H.-S.: Kim. H. R.: Ryu. E. K. Bull. Lonem Chem. Ser. 2001, 22, 135. (c) Familoni, O. B.; Kave, P. T.: Klaas. P. J. J. Chom. Sere. Chom. Commm 1998. 2563. (d) Bode. M. I.: Kave. P. T. J. Ch'm. Soc. Rorhin Trans. I 1993. 1809
4. (a) Jones. G. C'omprehensive Ileterocyclic (hemistry H: Katrikhy. A. R. Rees. C. W. Soriven. E. F. V. Eds. P'ergamon; Osford. 1996: Vol. 5. pp 167-243. (b) Balasubramanian. M.: Keay. J. G.

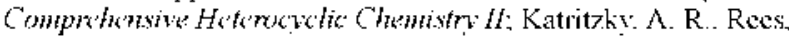
C. W. Seriven, F. F. V., F.ds.: Pergamon: Oxtord, 1996: Vol. 5. pp 245-300.

5. Lee. K. Y.: Kim. I. N. Bull Sorean (hem. Soc. 2002. 23. 939.

6. During the preparation of this manuscript. Basasaiah et al, have reported the similar results (Basasaish. D.: Reddy. R. M.: Kumaragumbaran. N.: Sharada. D. S. Tetrathedrom 2002. 58. 3693). They used iron and acetic acid for the syththesis of 3acetoxymethy-2( $1 H$-quindinones.

7. Benzisoxazoline 7: mp 168-169 " $\mathrm{C}$ : IR (KBr) $3277.1666 \mathrm{~cm}{ }^{~ '}:{ }^{\prime} \mathrm{H}$ NMR $\left(\mathrm{CWCl}_{3}\right) \delta 1.45(\mathrm{t} . J-7.1 \mathrm{H} z .3 \mathrm{H}) .2 .74(\mathrm{~s} .3 \mathrm{H}) .4 .40(\mathrm{q} . J-$ $7.1 \mathrm{~Hz} .2 \mathrm{H}) .7 .14$ (dd. $J-8.5$ and $2.0 \mathrm{~Hz} .1 \mathrm{H}) .7 .21(\mathrm{~d} . J-8.5 \mathrm{~Hz}$. III). 8.07 (d. $j-2.0 \mathrm{II}, 1 \mathrm{HI}$ ). $8.46(\mathrm{~s}, \mathrm{III}):{ }^{1.3} \mathrm{C}$ NMR $\left(\mathrm{CDCl}_{3}\right) \delta$ 14.12. 14.62, 59.32, 103.50. 111.95, 120.40, 121.81. 126.70. $128.43 .133 .41,145.99,165.75$ 